\title{
Does blood pressure variability contribute to risk stratification? Methodological issues and a review of outcome studies based on home blood pressure
}

\author{
Kei Asayama ${ }^{1,2}$, Fang-Fei Wei ${ }^{1,3}$, Yan-Ping Liu ${ }^{1}$, Azusa Hara ${ }^{1}$, Yu-Mei Gu ${ }^{1}$, Rudolph Schutte ${ }^{1,4}$, Yan Li $^{3}$, \\ Lutgarde Thijs ${ }^{1}$ and Jan A Staessen ${ }^{1,5}$
}

This review addresses methodological issues in the assessment of blood pressure variability and the predictive value of blood pressure variability derived from blood pressure readings obtained in the relaxed home environment. Preference should be given to indexes of blood pressure variability that are independent of the mean because we should evaluate the impact of blood pressure variability by eliminating the effect of blood pressure levels. Beat-to-beat blood pressure recordings outperform home blood pressure measurement in the assessment of blood pressure variability in longitudinal Belgian and Japanese population studies, whereas blood pressure variability did not incrementally predict outcome beyond blood pressure level and other cardiovascular risk factors. In conclusion, clinicians should focus on blood pressure level, given that it is the predominant risk factor and is manageable by lifestyle modifications and adequate antihypertensive drug treatment. Blood pressure variability remains a research tool that requires further prospective studies with hard end points to define its potential application, as it may be potentially useful in daily clinical practice.

Hypertension Research (2015) 38, 97-101; doi:10.1038/hr.2014.153; published online 16 October 2014

Keywords: average real variability; beat-to-beat blood pressure; blood pressure variability; home blood pressure; variability independent of the mean index

\section{INTRODUCTION}

Home blood pressure measurements have the advantage of being obtained in a relatively standardized and relaxed environment. Moreover, self-measurement is well accepted by patients and encourages active participation in the management of hypertension. ${ }^{1-5}$ Compared with conventional office blood pressure measurement, home blood pressure allows for a greater number of readings and minimizes the white coat effect, observer bias and measurement error, thereby contributing to enhanced diagnostic accuracy. Adjustment of antihypertensive medication based on home blood pressure readings might also reduce health-care costs. ${ }^{6}$ In addition, home blood pressure measurement offers several of the well-recognized advantages of the more complex approach of ambulatory blood pressure monitoring., ${ }^{4,7}$

Blood pressure variability has been a hot topic as some investigators have argued that stroke risk could be reduced more effectively by targeting systolic blood pressure variability along with systolic blood pressure level. ${ }^{8-10}$ We searched the PubMed database using 'blood pressure fluctuation' OR 'blood pressure instability' OR 'blood pressure variability' OR 'blood pressure variation' AND '1980/01/ 01'[Date-Publication]: '2014/05/30'[Date-Publication] as initial search terms, yielding 2066 publications. The number of publications was stable from 2010 to 2012 (113, 114 and 110 publications in 2010, 2011 and 2012, respectively). However, the number of publications increased to 175 in 2013. Similar to visit-to-visit variability in conventional clinic blood pressure measurements, ${ }^{9}$ multiple home blood pressure readings ${ }^{11-13}$ can provide information on blood pressure variability over varying time periods. The issue of whether naturally occurring blood pressure variability predicts risk beyond blood pressure level remains debated.

\section{ESTABLISHED AND NEWLY DEVELOPED INDEXES OF BLOOD PRESSURE VARIABILITY}

Short-term blood pressure variability measured on one occasion was reported in the early 1970s. ${ }^{14}$ In this report, Clement et al. ${ }^{14}$ assessed blood pressure variability using s.d. and coefficient of variation (CV) values obtained every $5 \mathrm{~min}$ for $3 \mathrm{~h}$ in 70 untreated hypertensive

\footnotetext{
${ }^{1}$ Studies Coordinating Centre, Research Unit Hypertension and Cardiovascular Epidemiology, KU Leuven Department of Cardiovascular Sciences, University of Leuven, Leuven, Belgium; ${ }^{2}$ Department of Planning for Drug Development and Clinical Evaluation, Tohoku University Graduate School of Pharmaceutical Sciences, Sendai, Japan; ${ }^{3}$ Center for Epidemiological Studies and Clinical Trials and Center for Vascular Evaluations, Shanghai Key Laboratory of Hypertension, Shanghai Institute of Hypertension, Ruijin Hospital, Shanghai Jiaotong University School of Medicine, Shanghai, China; ${ }^{4}$ Hypertension in Africa Research Team (HART), North-West University, Potchefstroom, South Africa and ${ }^{5}$ VitaK Research and Development, Maastricht University, Maastricht, The Netherlands

Correspondence: Dr K Asayama, Studies Coordinating Centre, Research Unit Hypertension and Cardiovascular Epidemiology, KU Leuven Department of Cardiovascular Sciences, University of Leuven, Campus Sint Rafaël, Kapucijnenvoer 35, Block D, Box 7001, Leuven 3000, Belgium.

E-mail: kei@asayama.org

Received 9 January 2014; revised 30 July 2014; accepted 19 August 2014; published online 16 October 2014
} 
patients. These researchers identified a positive association between sympathetic activity and the s.d. of blood pressure. However, blood pressure level and s.d. were correlated; thus, the correlations of variability with the indexes of sympathetic activity disappeared when variability was expressed as CV. In the early 1980s, Mancia ${ }^{15}$ performed continuous intravascular recordings for $24 \mathrm{~h}$ in 25 untreated hypertensive patients. This study revealed that the s.d. was significantly reduced after antihypertensive drug intervention, whereas the CV was not. ${ }^{15}$ Nevertheless, the results can still be influenced by the blood pressure level. ${ }^{8,9}$

Mena et al. ${ }^{16}$ proposed average real variability (ARV) as a novel index representing short-tem, reading-to-reading, within-subject variability in blood pressure. ARV attempts to correct for the limitations of the commonly used s.d., which accounts only for the dispersion of values around the mean and not for the order of the blood pressure readings. ${ }^{17,18} \mathrm{ARV}$ is calculated using the following formula:

$$
\mathrm{ARV}=\frac{1}{\sum \mathrm{w}_{k}} \sum_{k=1}^{n-1} \mathrm{w}_{k} \times\left|\mathrm{BP}_{k+1}-\mathrm{BP}_{\mathrm{k}}\right|
$$

where $n$ is the number of blood pressure readings, $k$ ranges from 1 to $n-1$ and $w_{k}$ is the time interval between $\mathrm{BP}_{k}$ and $\mathrm{BP}_{k+1}$. ARV therefore accounts for the order of the measurements and weighs each value according to the measurement interval. However, ARV remains correlated with the blood pressure levels.

Rothwell et al. ${ }^{8,9}$ proposed blood pressure variability independent of the mean (VIM) as a new index, which might serve as a better predictor of cardiovascular outcome. VIM is calculated as the s.d. divided by the mean to the power $x$ and multiplied by the population mean to the power $x$. The power $x$ is obtained by fitting a curve through a plot of s.d. against mean using the model s.d. $=a \times$ mean $^{x}$, where $x$ is derived by nonlinear regression analysis. Because VIM is derived from the distribution of the blood pressure values in each population, VIM is tied to the population being examined and cannot be compared across the population. A strong correlation is noted for VIM and other indexes of blood pressure. ${ }^{19}$ Nevertheless, VIM does not correlate with blood pressure levels, ${ }^{8,9}$ assuring that we are able to evaluate the impact of blood pressure variability using VIM by eliminating the effect of blood pressure levels.

The concept of blood pressure variability is wide ranging and extends beyond reading-to-reading variability. The diurnal blood pressure profile normally includes a $10-20 \%$ fall in blood pressure during sleep, which is driven by physical inactivity and mostly independent of an endogenous rhythm. ${ }^{20}$ Based on the International Database on Ambulatory blood pressure monitoring in relation to Cardiovascular Outcomes (IDACO) project, we reported that after adjustment for cardiovascular risk factors and the 24-h blood pressure, the night-to-day ratio predicted total, cardiovascular and noncardiovascular mortality, but not fatal combined with nonfatal cardiovascular events. ${ }^{21}$ Kario et al. ${ }^{22}$ proposed the definitions of the sleeptrough and preawakening morning surge in blood pressure, which are independent predictors of stroke in elderly Japanese hypertensive patients. Their findings were supported by the Ohasama study involving 1430 individuals from the general population, which revealed that these factors predicted cerebral hemorrhage (quintile trend $P=0.04$ ) but not ischemic stroke (quintile trend $P=0.94$ ). However, Verdecchia et al. ${ }^{23}$ reported that neither patients with a high sleep-trough $(>36.0 \mathrm{~mm} \mathrm{Hg})$ nor those with a high preawakening ( $>27.5 \mathrm{~mm} \mathrm{Hg}$ ) systolic pressure had independent risks for mortality and cardiovascular events. The IDACO analysis demonstrated that an exaggerated morning surge ( $\geqslant 90$ th percentile of the population) is an independent risk factor for mortality as well as cardiovascular and cardiac events, whereas a sleep-trough or preawakening morning surge in systolic blood pressure $<20 \mathrm{~mm} \mathrm{Hg}$ is not. ${ }^{24}$ All of these heterogeneous forms of blood pressure variation reflect different biological phenomena that exhibit low correlation among these processes. ${ }^{25}$

\section{VARIABILITY OF HOME BLOOD PRESSURE MEASURED BY OBSERVERS}

The clinical usefulness of blood pressure variability beyond blood pressure levels should be evaluated by assessing hard outcomes. Based on the Flemish Study on Environment, Genes and Health Outcomes (FLEMENGHO) study, ${ }^{26}$ we investigated the predictive value of home blood pressure variability in 2944 participants (mean age, 44.9 years; $50.7 \%$ women). ${ }^{27}$ At baseline, trained nurses measured each participant's blood pressure at two home visits during an interval of 2-4 weeks. At each home visit, the nurses obtained five consecutive blood pressure readings to the nearest $2 \mathrm{~mm} \mathrm{Hg}$ using mercury sphygmomanometers after the participants rested for $5 \mathrm{~min}$ in the sitting position. A stringent program for quality assurance and quality control was implemented. ${ }^{28}$ Every 3 months, the observers were required to pass a test that involved reading blood pressures from a videotape featuring a falling mercury column with Korotkoff sounds. Their readings were required to comply within $5 \mathrm{~mm} \mathrm{Hg}$ of those of senior medical staff. Digit preference was assessed at 6-month intervals.

We assessed within-subject overall (10 readings), within- and between-visit systolic blood pressure variability using VIM, maximum minus minimum blood pressure difference (MMD) and ARV. During a median follow-up of 12.3 years (5th to 95th percentile interval, 2.7-23.7 years), 401 deaths occurred, and 311 participants experienced a fatal or nonfatal cardiovascular event. Overall systolic blood pressure variability averaged $5.45 \mathrm{U}, 15.87 \mathrm{~mm} \mathrm{Hg}$ and $4.08 \mathrm{~mm} \mathrm{Hg}$ for VIM, MMD and ARV, respectively. Women, older age, higher systolic blood pressure, lower body mass index, a history of peripheral arterial disease and use of $\beta$-blockers were the main correlates of systolic blood pressure variability. In multivariable-adjusted analyses, including both systolic blood pressure and a measure of variability, systolic blood pressure predicted the outcomes, whereas any measure of variability did not. To assess the additional risk explained in the Cox regression model, we applied the generalized $R^{2}$ statistic ${ }^{29}$ by adding the indices of blood pressure variability to models already including the systolic blood pressure level and covariables; the $R^{2}$ statistics were $\leqslant 0.10 \%$. The 10-year multivariable-adjusted risk of a composite cardiovascular event increased with systolic blood pressure $(P<0.0001)$ but not with overall VIM $(P=0.31)$, and the results for the risk of death were similar. These findings suggest that within-subject blood pressure variability measured by an observer does not exhibit any prognostic significance beyond systolic pressure levels in the general population.

\section{VARIABILITY OF SELF-MEASURED HOME BLOOD PRESSURE}

Several Japanese groups have assessed the relation between targetorgan damage or the incidence of cardiovascular complications and blood pressure variability as captured by self-measurements at home. Ushigome et al..$^{30}$ demonstrated significant correlations between macroalbuminuria in type 2 diabetes mellitus and the CV of home blood pressure readings. Using a multivariable-adjusted linear regression analysis including blood pressure level, these authors recently reported that pulse wave velocity was significantly correlated with the s.d. of three consecutive measurements on one occasion in the morning $(P=0.016)$ or evening $(P=0.0099)$ but not with the s.d. of 
day-to-day home blood pressure readings $(P \geqslant 0.78) .{ }^{31}$ Nishimura et al. $^{32}$ demonstrated that a low estimated glomerular filtration rate was significantly associated with the s.d. of home blood pressure readings. Researchers have placed expectations on home blood pressure variability as a biomarker for chronic kidney disease. ${ }^{33}$ However, as reported by Okada et al., ${ }^{34}$ s.d., CV and ARV of selfmeasured home blood pressure readings did not predict the progression of chronic kidney disease.

The prognostic significance of self-measured home day-to-day blood pressure variability was first assessed in the Ohasama population using the within-participant s.d. of morning systolic blood pressure readings over 26 days (median). ${ }^{13}$ In multivariable-adjusted Cox models also including blood pressure level, the s.d. was associated with higher risks of total mortality (hazard ratio (HR) per 1 s.d. increase in the within-participant s.d., 1.18; 95\% confidence intervals, 1.07-1.31), cardiovascular mortality (HR, 1.20; CI, 1.02-1.40), noncardiovascular mortality (HR, 1.18; CI, 1.04-1.34) and stroke mortality (HR, 1.38; CI, 1.12-1.72). The association was not observed for cardiac mortality (HR, 1.02; CI, 0.89-1.29). The association of blood pressure variability with non-cardiovascular mortality was difficult to interpret but might reflect reverse causality (i.e., subclinical disease leading to greater variability). The Finn-Home investigators assessed the day-to-day variability of the self-measured systolic blood pressure readings in the morning. The within-participant s.d. over 7 days predicted total mortality and cardiovascular events. ${ }^{35}$ The HRs for an 1-s.d. increment in variability $(3.93 \mathrm{~mm} \mathrm{Hg}$ ) were 1.17 (CI, 1.00-1.30; $P=0.03$ ) and 1.21 (CI, 1.08-1.40; $P=0.006$ ), respectively. ${ }^{35}$ Day-today variability in the evening systolic blood pressure readings was not predictive $(P \geqslant 0.11) .{ }^{35}$

Based on the self-measured home blood pressure readings, we explored whether fatal and nonfatal outcomes were associated with the new indexes of blood pressure variability. ${ }^{7}$ We analyzed mortality and stroke risk in 2421 Ohasama residents after excluding high-risk participants with a history of stroke. In the Ohasama study, physicians and public health nurses instructed participants how to measure their home blood pressure using a validated ${ }^{36}$ oscillometric device (OMRON HEM 401C; Omron Healthcare, Kyoto, Japan). Participants were asked to record their blood pressure for 4 weeks after at least $2 \mathrm{~min}$ of rest in the morning within $1 \mathrm{~h}$ after awakening and, if applicable, before taking their blood pressure-lowering medications. Participants also obtained the recordings in the evening, immediately before going to bed. We assessed the independent predictive value of the within-subject mean systolic blood pressure and corresponding variability indexes, namely VIM, MMD and ARV. Over a median follow-up of 12.0 years, 412 participants died with any cause, 139 attributed to cardiovascular causes death and 223 participants had a stroke. In multivariable-adjusted Cox models including the morning systolic pressure and other covariates, VIM and ARV predicted total and cardiovascular mortality in all participants $(P \leqslant 0.044)$, whereas VIM predicted cardiovascular mortality in treated $(P=0.014)$ but not in untreated $(P=0.23)$ participants. Morning MMD was not predictive of any end points $(P \geqslant 0.085)$. In models that already included evening systolic pressure, only VIM predicted cardiovascular mortality in all patients as well as untreated participants $(P \leqslant 0.046)$. Both morning and evening systolic blood pressure were consistent predictors $(P \leqslant 0.032)$ with the exception of cardiovascular mortality in treated participants for morning systolic pressure $(P=0.082)$ when we calculated the multivariable-adjusted 10-year risk of cardiovascular mortality and stroke incidence in relation to the mean level and VIM (Figure 1 in all of the participants). The $R^{2}$ statistic, ${ }^{29}$ which was also calculated in the above-mentioned FLEMENGHO population, ${ }^{27}$ ranged from $<0.01$ to $0.88 \%$. Being on antihypertensive drug treatment appears to be the main driver of the significant associations between cardiovascular mortality and blood pressure variability. ${ }^{7}$ In the Japanese population sample, the new indexes of blood pressure

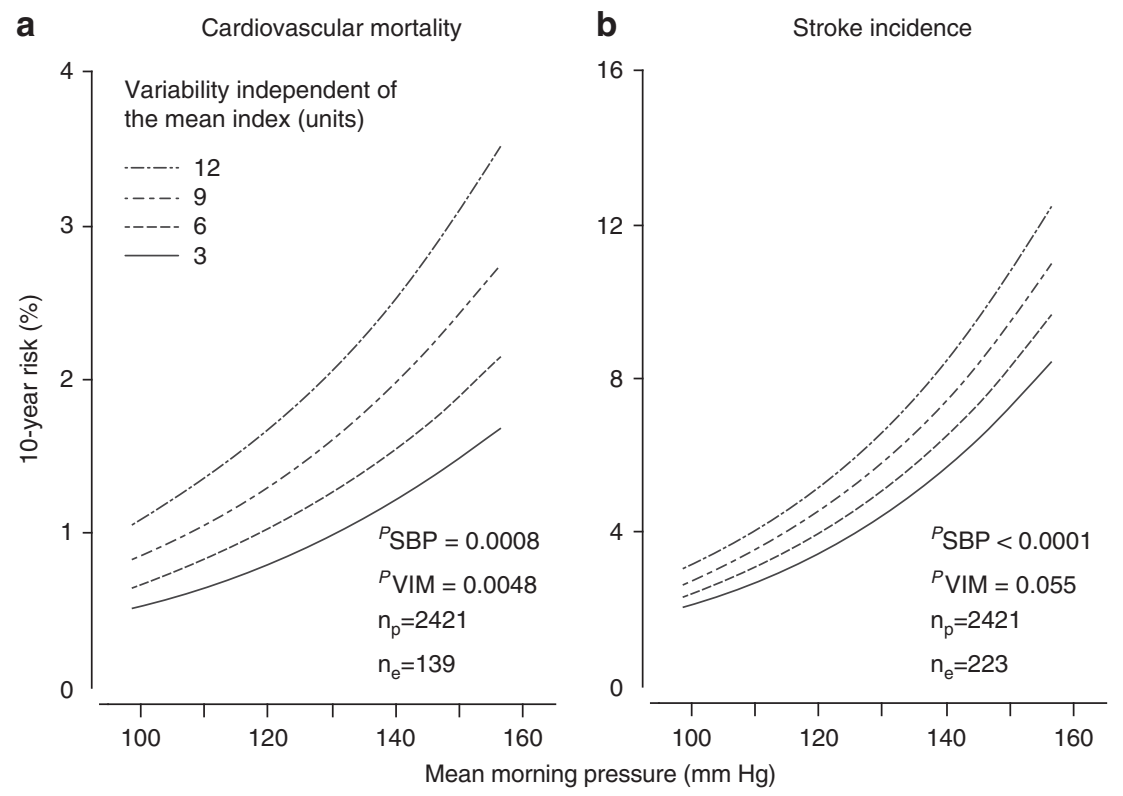

Figure 1 Absolute 10-year risk of cardiovascular mortality (a) and stroke incidence (b) in relation to the mean level of systolic blood pressure measured at home in the morning in 2421 participants. The analyses were standardized to the distributions (mean or ratio) of sex, age, body mass index, heart rate, smoking and drinking, total cholesterol, diabetes mellitus, history of cardiovascular diseases and treatment with antihypertensive drugs. Four continuous lines equal to 3, 6, 9 and $12 \mathrm{U}$ represent the risk that is independently associated with VIM. $P$-values represent the independent effects of SBP ( $P_{\mathrm{SBP}}$ ) and VIM $\left(P_{\mathrm{VIM}}\right) . n_{\mathrm{p}}$ and $n_{\mathrm{e}}$ indicate the number of participants at risk and the number of events. Reproduced from Asayama et al. ${ }^{7}$ 
a

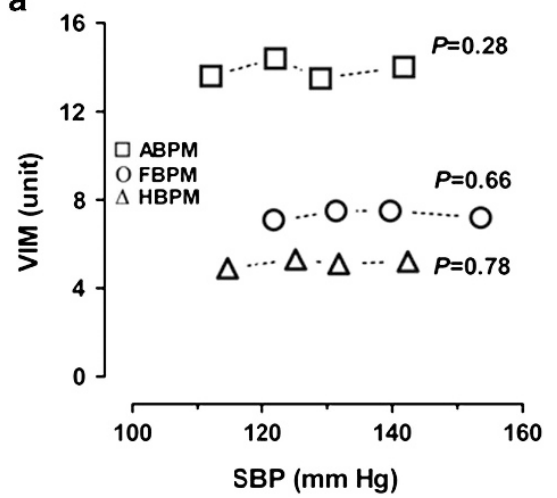

b

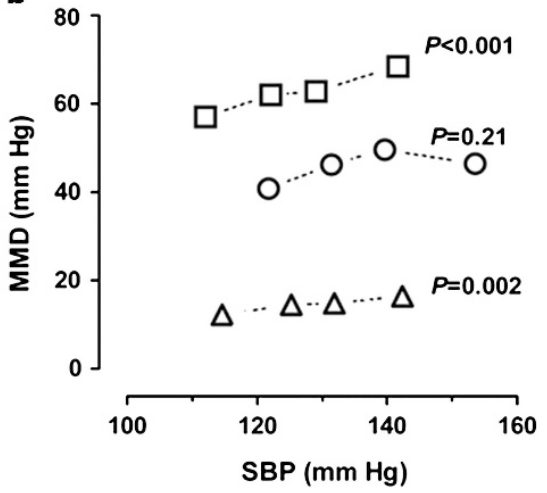

C

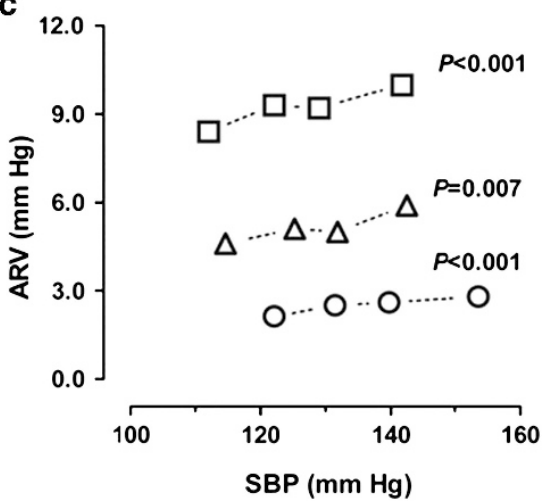

Figure 2 Variability independent of the mean (VIM; a), maximum-minimum difference (MMD; b) and average real variability (ARV; c) by quartiles of the distribution of the mean systolic blood pressure (SBP) for 24-h ( $\square$ ), home $(\bigcirc)$ or beat-to-beat $(\Delta)$ measurements. $P$-values represent the linear trends across the quartiles of SBP. Reproduced from Wei et al. ${ }^{39}$

variability derived from self-measured home blood pressure readings did not incrementally predict outcome beyond mean systolic pressure.

\section{VARIABILITY DERIVED FROM BEAT-TO-BEAT BLOOD PRESSURE READING}

Few studies have addressed the risk associated with blood pressure variability as captured by beat-to-beat recordings. Parati et al. ${ }^{37}$ recorded continuous intra-arterial blood pressure for $24 \mathrm{~h}$ in 108 untreated hospitalized patients (mean age, 43.8 years; $44.4 \%$ women). Target-organ damage was assessed from the electrocardiographic Sokolow-Lyon index, radiographic cardiac volume, Keith-Wagener's classification of retinal lesions and a history of cardiovascular or renal complications. In analyses by quintiles of the 24-h continuous blood pressure level, patients whose blood pressure variability was below average in each quintile had a lower prevalence and severity of targetorgan damage than those with a higher than average blood pressure variability. ${ }^{37}$ Veerman et al. ${ }^{38}$ studied 33 hypertensive patients (mean age, 41 years; $48.5 \%$ women) who were off treatment for at least 3 months. Blood pressure was recorded using the Finapres Model 5 for 20 min with participants in the supine position. The logarithmically transformed urinary albumin-to-creatinine ratio correlated with diastolic $(r=0.37 ; P=0.037)$ but not with systolic blood pressure variability as measured by the s.d. ${ }^{38}$ The associations of left ventricular mass index with systolic and diastolic blood pressure variability were not significant. ${ }^{38}$ The drawbacks of these previous studies ${ }^{37,38}$ include the use of s.d. as a measure of blood pressure variability, ${ }^{37}$ small sample size, ${ }^{37,38}$ selection of hospitalized participant ${ }^{37}$ and the use of dichotomized instead of continuous variability indexes in multivariable-adjusted analyses. ${ }^{37}$

Recently, Wei et al. ${ }^{39}$ assessed target-organ damage markers and new indexes of blood pressure variability based on 10-min beat-tobeat, 24-h ambulatory and 7-day home blood pressure recordings among 256 Chinese outpatients (mean age, 51.1 years) who were all off blood pressure-lowering treatment. The patients' beat-to-beat finger blood pressures were recorded for $10 \mathrm{~min}$ after participants rested for at least $10 \mathrm{~min}$ in the supine position. The recordings were obtained with the Finometer PRO device (Finapres Medical System BV, Amsterdam, The Netherlands) using the volume-clamp method. ${ }^{40}$ Target-organ damage was assessed from aortic pulse wave velocity, left ventricular hypertrophy and the urinary albumin-to-creatinine ratio. In unadjusted categorical analyses across quartiles of mean systolic blood pressure levels (Figure 2), VIM did not correlate with pressure level $(P \geqslant 0.28)$ regardless of the type of blood pressure recording.
With the exception of the MMD in beat-to-beat recordings $(P=0.21)$, MMD and ARV positively correlated with pressure level $(P \leqslant 0.007)$ across quartiles of systolic blood pressure. In multiple regression analysis, left ventricular mass index was not associated with mean systolic pressure levels in beat-to-beat recordings $(P \geqslant 0.18)$ but was positively associated $(P<0.038)$ with VIM $\left(+3.144 \mathrm{~g} \mathrm{~m}^{-2}\right)$, MMD $\left(+3.528 \mathrm{~g} \mathrm{~m}^{-2}\right)$ and ARV $\left(+2.968 \mathrm{~g} \mathrm{~m}^{-2}\right)$ independently of systolic blood pressure and other covariables. The urinary albumin-tocreatinine ratio positively associated $(P \leqslant 0.03)$ with the level of mean systolic blood pressure $\left(+1.169 \mathrm{mg} \mathrm{mmol}^{-1}\right)$ and MMD $(+1.183$ $\mathrm{mg} \mathrm{mmol}^{-1}$ ), and pulse wave velocity only associated with the level of systolic pressure $\left(+0.689 \mathrm{~m} \mathrm{~s}^{-1} ; \quad P<0.001\right)$. We assessed the variance inflation factor in all models including both level and variability of beat-to-beat blood pressure. The variance inflation factor did not exceed 1.24, indicating minimal collinearity. In 24-h and home blood pressure recordings, the indexes of target-organ damage positively associated $(P<0.05)$ with the level of pressure but were not consistently associated with the indexes of blood pressure variability. Beat-to-beat recordings may therefore outperform home and 24-h ambulatory blood pressure monitoring in the assessment of targetorgan damage in relation to blood pressure variability. The opposite might be true for evaluating the contribution of blood pressure levels to target-organ damage. However, only surrogate end points in crosssectional studies have been assessed for association with level and variability in beat-to-beat blood pressure recordings. ${ }^{37,38}$ Only one prospective study addressed the incidence of cardiovascular complications in relation to blood pressure levels as assessed by home invasive intra-arterial 24-h ambulatory recordings in hypertensive patients, but this study did not provide information regarding blood pressure variability. ${ }^{41,42}$ Based on the findings from the Northwick Park Heart Study, ${ }^{39,40}$ one might speculate that the ability of beat-to-beat recordings to demonstrate associations with targetorgan damage might depend on the recording technique (intra-arterial vs. finger plethysmography) and the duration of the recordings (10 min vs. $24 \mathrm{~h}$ ).

\section{CONCLUSION}

Blood pressure variability should preferably be assessed by indexes that are independent of blood pressure level, such as VIM. ${ }^{8,9}$ Even when we observe a significant prognostic effect of s.d. or other variability indexes, we cannot conclude the usefulness of blood pressure variability because it is affected by the level. However, recent studies of home blood pressure variability as captured by VIM failed to 
demonstrate that blood pressure variability substantially refines risk profiling beyond blood pressure levels; ${ }^{7,27}$ therefore, it is less meaningful to consider variability indexes for risk stratification. Blood pressure level, the predominant risk factor manageable by lifestyle modifications and adequate antihypertensive drug treatment ${ }^{43}$ should remain the primary focus for risk stratification and treatment in clinical practice. Blood pressure variability currently remains a research tool that requires further prospective studies with hard end points to define potential applications, where it might be useful in daily clinical practice.

1 Asayama K, Ohkubo T, Kikuya M, Metoki H, Obara T, Hoshi H, Hashimoto J, Totsune K, Satoh H, Imai Y. Use of 2003 European Society of Hypertension-European Society of Cardiology guidelines for predicting stroke using self-measured blood pressure at home: the Ohasama study. Eur Heart J 2005; 26: 2026-2031.

2 Little P, Barnett J, Barnsley L, Marjoram J, Fitzgerald-Barron A, Mant D. Comparison of acceptability of and preferences for different methods of measuring blood pressure in primary care. BMJ 2002; 325: 258-259.

3 Ohkubo T, Imai Y, Tsuji I, Nagai K, Kato J, Kikuchi N, Nishiyama A, Aihara A, Sekino M, Kikuya M, Ito S, Satoh H, Hisamichi S. Home blood pressure measurement has a stronger predictive power for mortality than does screening blood pressure measurement: a population-based observation in Ohasama, Japan. J. Hypertens 1998; 16: 971-975.

4 Asayama $\mathrm{K}$, Ohkubo $\mathrm{T}$, Kikuya $\mathrm{M}$, Obara $\mathrm{T}$, Metoki $\mathrm{H}$, Inoue $\mathrm{R}$, Hara $\mathrm{A}$, Hirose $\mathrm{T}$, Hoshi H, Hashimoto J, Totsune K, Satoh H, Imai Y. Prediction of stroke by home 'morning' versus 'evening' blood pressure values: the Ohasama study. Hypertension 2006; 48: 737-743.

5 Niiranen TJ, Hanninen MR, Johansson J, Reunanen A, Jula AM. Home-measured blood pressure is a stronger predictor of cardiovascular risk than office blood pressure: the Finn-Home study. Hypertension 2010; 55: 1346-1351.

6 Staessen JA, Den Hond E, Celis H, Fagard R, Keary L, Vandenhoven G, O'Brien ET. Antihypertensive treatment based on blood pressure measurement at home or in the physician's office: a randomized controlled trial. JAMA 2004; 291: 955-964.

7 Asayama K, Kikuya M, Schutte R, Thijs L, Hosaka M, Satoh M, Hara A, Obara T, Inoue R, Metoki H, Hirose T, Ohkubo T, Staessen JA, Imai Y. Home blood pressure variability as cardiovascular risk factor in the population of Ohasama. Hypertension 2013; 61: 61-69.

8 Rothwell PM, Howard SC, Dolan E, O'Brien E, Dobson JE, Dahlof B, Poulter NR, Sever PS. Effects of beta blockers and calcium-channel blockers on within-individual variability in blood pressure and risk of stroke. Lancet Neurol 2010; 9: 469-480.

9 Rothwell PM, Howard SC, Dolan E, O'Brien E, Dobson JE, Dahlof B, Sever PS, Poulter NR. Prognostic significance of visit-to-visit variability, maximum systolic blood pressure, and episodic hypertension. Lancet 2010; 375: 895-905.

10 Webb AJ, Fischer U, Mehta Z, Rothwell PM. Effects of antihypertensive-drug class on interindividual variation in blood pressure and risk of stroke: a systematic review and meta-analysis. Lancet 2010; 375: 906-915.

11 Johansson JK, Niiranen TJ, Puukka PJ, Jula AM. Factors affecting the variability of home-measured blood pressure and heart rate: the Finn-Home study. J Hypertens 2010; 28: 1836-1845.

12 Kato T, Kikuya M, Ohkubo T, Satoh M, Hara A, Obara T, Metoki H, Asayama K, Hirose T, Inoue R, Kanno A, Totsune K, Hoshi H, Satoh H, Imai Y. Factors associated with day-by-day variability of self-measured blood pressure at home: the Ohasama study. Am J Hypertens 2010; 23: 980-986.

13 Kikuya M, Ohkubo T, Metoki H, Asayama K, Hara A, Obara T, Inoue R, Hoshi H, Hashimoto J, Totsune K, Satoh H, Imai Y. Day-by-day variability of blood pressure and heart rate at home as a novel predictor of prognosis: the Ohasama study. Hypertension 2008; 52: 1045-1050.

14 Clement DL, Mussche MM, Vanhoutte G, Pannier R. Is blood pressure variability related to activity of the sympathetic system? Clin Sci 1979; 57 (Suppl 5): $217 \mathrm{~s}-219 \mathrm{~s}$.

15 Mancia G. Blood pressure variability at normal and high blood pressure. Chest 1983; 83: 317-320.

16 Mena L, Pintos S, Queipo NV, Aizpurua JA, Maestre G, Sulbaran T. A reliable index for the prognostic significance of blood pressure variability. J Hypertens 2005; 23 : 505-511.

17 Hansen TW, Thijs L, Li Y, Boggia J, Kikuya M, Björklund-Bodegård K, Richart T, Ohkubo T, Jeppesen J, Torp-Pedersen C, Dolan E, Kuznetsova T, Stolarz-Skrzypek K, Tikhonoff V, Malyutina S, Casiglia E, Nikitin Y, Lind L, Sandoya E, Kawecka-Jaszcz K, Imai Y, Wang J, Ibsen H, O'Brien E, Staessen JA. Prognostic value of reading-to-reading blood pressure variability over $24 \mathrm{~h}$ in 8938 subjects from 11 populations. Hypertension 2010; 55: 1049-1057.

18 Parati G, Rizzoni D. Assessing the prognostic relevance of blood pressure variability: discrepant information from different indices. J Hypertens 2005; 23: 483-486.

19 Levitan EB, Kaciroti N, Oparil S, Julius S, Muntner P. Relationships between metrics of visit-to-visit variability of blood pressure. J Hum Hypertens 2013; 27: 589-593.
20 Baumgart P, Walger P, Fuchs G, Dorst KG, Vetter H, Rahn KH. Twenty-four-hour blood pressure is not dependent on endogenous circadian rhythm. J Hypertens 1989; 7: 331-334.

21 Fan HQ, Li Y, Thijs L, Hansen TW, Boggia J, Kikuya M, Björklund-Bodegård K, Richart T, Ohkubo T, Jeppesen J, Torp-Pedersen C, Dolan E, Kuznetsova T, Stolarz-Skrzypek K, Tikhonoff V, Malyutina S, Casiglia E, Nikitin Y, Lind L, Sandoya E, Kawecka-Jaszcz K, Imai Y, Ibsen H, O'Brien E, Wang J, Staessen JA, International Database on Ambulatory Blood Pressure In Relation to Cardiovascular Outcomes Investigators. Prognostic value of isolated nocturnal hypertension on ambulatory measurement in 8711 individuals from 10 populations. J Hypertens 2010; 28: 2036-2045

22 Kario K, Pickering TG, Umeda Y, Hoshide S, Hoshide Y, Morinari M, Murata M, Kuroda T, Schwartz JE, Shimada K. Morning surge in blood pressure as a predictor of silent and clinical cerebrovascular disease in elderly hypertensives: a prospective study. Circulation 2003; 107: 1401-1406.

23 Verdecchia P, Angeli F, Mazzotta G, Garofoli M, Ramundo E, Gentile G, Ambrosio G, Reboldi G. Day-night dip and early-morning surge in blood pressure in hypertension: prognostic implications. Hypertension 2012; 60: 34-42.

$24 \mathrm{Li} \mathrm{Y,} \mathrm{Thijs} \mathrm{L,} \mathrm{Hansen} \mathrm{TW,} \mathrm{Kikuya} \mathrm{M,} \mathrm{Boggia} \mathrm{J,} \mathrm{Richart} \mathrm{T,} \mathrm{Metoki} \mathrm{H,} \mathrm{Ohkubo} \mathrm{T,}$ Torp-Pedersen C, Kuznetsova T, Stolarz-Skrzypek K, Tikhonoff V, Malyutina S, Casiglia E, Nikitin Y, Sandoya E, Kawecka-Jaszcz K, Ibsen H, Imai Y, Wang J, Staessen JA. Prognostic value of the morning blood pressure surge in 5645 subjects from 8 populations. Hypertension 2010; 55: 1040-1048.

25 Muntner P, Shimbo D, Diaz KM, Newman J, Sloan RP, Schwartz JE. Low correlation between visit-to-visit variability and $24-\mathrm{h}$ variability of blood pressure. Hypertens. Res. 2013; 36: 940-946.

26 Staessen JA, Wang JG, Brand E, Barlassina C, Birkenhager WH, Herrmann SM, Fagard R, Tizzoni L, Bianchi G. Effects of three candidate genes on prevalence and incidence of hypertension in a Caucasian population. J Hypertens 2001; 19: 1349-1358.

27 Schutte R, Thijs L, Liu YP, Asayama K, Jin Y, Odili A, Gu YM, Kuznetsova T, Jacobs L, Staessen JA. Within-subject blood pressure level—not variability-predicts fatal and nonfatal outcomes in a general population. Hypertension 2012; 60: 1138-1147.

28 Staessen J, Bulpitt CJ, Fagard R, Joossens JV, Lijnen P, Amery A. Familial aggregation of blood pressure, anthropometric characteristics and urinary excretion of sodium and potassium-a population study in two Belgian towns. J Chronic Dis 1985; 38: 397-407.

29 Cox DR, Snell EJ. Analysis of Binary Data, 2nd edn. Chapman and Hall/CRC: Boca Raton, FL, USA, 1989

30 Ushigome E, Fukui M, Hamaguchi M, Senmaru T, Sakabe K, Tanaka M, Yamazaki M, Hasegawa G, Nakamura N. The coefficient variation of home blood pressure is a novel factor associated with macroalbuminuria in type 2 diabetes mellitus. Hypertens Res 2011; 34: 1271-1275.

31 Fukui M, Ushigome E, Tanaka M, Hamaguchi M, Tanaka T, Atsuta H, Ohnishi M, Oda Y, Hasegawa G, Nakamura N. Home blood pressure variability on one occasion is a novel factor associated with arterial stiffness in patients with type 2 diabetes. Hypertens Res 2013; 36: 219-225.

32 Nishimura M, Kato Y, Tanaka T, Todo R, Tone A, Yamada K, Ootani S, Kawabe Y, Yoshizumi H, Hoshiyama $Y$. Significance of estimating the glomerular filtration rate for the management of hypertension in type 2 diabetes with microalbuminuria. Hypertens Res 2013; 36: 705-710.

33 Romero MJ. Home blood pressure variability: a new target to monitor in chronic kidney disease patients with low eGFR? Hypertens Res 2013; 36: 673-675.

34 Okada T, Matsumoto H, Nagaoka Y, Nakao T. Association of home blood pressure variability with progression of chronic kidney disease. Blood Press Monit 2012; 17: $1-7$.

35 Johansson JK, Niiranen TJ, Puukka PJ, Jula AM. Prognostic value of the variability in home-measured blood pressure and heart rate: the Finn-Home study. Hypertension 2012; 59: 212-218.

36 Imai Y, Abe K, Sekino H. Evaluation of automatic devices for blood pressure measurement. Annu Rev Nephrol 1992; 1991: 55-61.

37 Parati G, Pomidossi G, Albini F, Malaspina D, Mancia G. Relationship of 24-hour blood pressure mean and variability to severity of target-organ damage in hypertension. J Hypertens 1987; 5: 93-98.

38 Veerman DP, de Blok K, van Montfrans A. Relationship of steady state and ambulatory blood pressure variability to left ventricular mass and urinary albumin excretion in essential hypertension. Am J Hypertens 1996; 9: 455-460.

39 Wei FF, Li Y, Zhang L, Xu TY, Ding FH, Wang JG, Staessen JA. Beat-to-beat, reading-toreading, and day-to-day blood pressure variability in relation to organ damage in untreated Chinese. Hypertension 2014; 63: 790-796.

40 Penaz J. Criteria for set point estimation in the volume clamp method of blood pressure measurement. Physiol Res 1992; 41: 5-10.

41 Acharya DU, Heber ME, Dore CJ, Raftery EB. Ambulatory intraarterial blood pressure in essential hypertension. Effects of age, sex, race, and body mass - the Northwick Park Hospital Database Study. Am J Hypertens 1996; 9: 943-952.

42 Khattar RS, Swales JD, Banfield A, Dore C, Senior R, Lahiri A. Prediction of coronary and cerebrovascular morbidity and mortality by direct continuous ambulatory blood pressure monitoring in essential hypertension. Circulation 1999; 100: 1071-1076.

43 Asayama K, Ohkubo T, Metoki H, Obara T, Inoue R, Kikuya M, Thijs L, Staessen JA, Imai Y. Cardiovascular outcomes in the first trial of antihypertensive therapy guided by self-measured home blood pressure. Hypertens Res 2012; 35: 1102-1110. 